\title{
Control of Poly(styrene) Particles Size by In-Situ Polymerization
}

\author{
Shigeki ChuJo, Masayoshi Rikukawa, Kohei Sanui, Naoya OGata, ${ }^{*}{ }^{\dagger}$ \\ Satoshi KoIZumi, ${ }^{* *}$ and Takeji HASHIMOTO $* * *$ \\ Department of Chemistry, Sophia University, 7-1 Kioi-cho, Chiyoda-ku, Tokyo 102-0094, Japan \\ ${ }^{*}$ Department of Photonic Material Science, Chitose Institute of Science and Technology, \\ 758-65 Bibi, Chitose, Hokkaido 066-8655, Japan \\ **Advanced Research Center, Japan Atomic Energy Research Institute, \\ 2-4 Shirakatashirane, Tokaimura, Ibaraki-ken 319-1100, Japan \\ *** Department of Polymer Chemistry, Graduate School of Engineering, Kyoto University, \\ Yoshidahonmachi, Sakyo-ku, Kyoto 606-8317, Japan
}

(Received June 7, 1999; Accepted July 31, 2000)

\begin{abstract}
Styrene (St) was polymerized in mixed solvents of ethanol $/ n$-hexane as good-poor solvents for matrix polymers such as poly(methylvinylether) (PMVE) in solution. Conformational change of the matrix polymer from extended to random coil structures provided different dimensional fields for in-situ polymerization of St, resulting in control of particle size and distribution of poly(St) (PSt). Neutron scattering of St solution suggested the formation of St monomer clusters in matrix polymer solution.

KEY WORDS Styrene / In-situ Polymerization / Matrix Polymers / Microspheres / Monomer Cluster / Neutron Scattering /
\end{abstract}

Emulsion or suspension polymerization of olefine monomers such as styrene (St) are used to prepare fine particles in water as medium. Normally, emulsion polymerization is carried out using an emulsifying agent such as a surfactant in the presence of water-soluble radical initiators, while suspension polymerization is carried out in aqueous solution of water-soluble polymers such as poly(vinylalcohol) (PVA) to disperse olefine monomers in water in the presence of water-insoluble radical initiators. PVA as dispersing agent for the suspension polymerization increases solution viscosity to stabilize the monomer domain in aqueous solution. Control of particle size or size distribution of resulting olefine polymers is not so easy from the standpoint of process control. ${ }^{1}$

Non-aqueous solvents such as alcohol are used to obtain fine particles of olefine polymers not dissolved in non-aqueous solvents. Fine particles of olefine polymers are used for dry coating or toner for copy processes. In 1982, microspheres of PSt by dispersion polymerization in alcoholic solvents were prepared. It was diffucult by this process to obtain monodispersed size distribution of PSt particles. We previously studied in-situ polymerization to prepare nano-composite materials from commodity polymers with liquid crystalline polymers (LCP). ${ }^{2}$ Insitu polymerization for nano-composites was carried out by polymerizing liquid crystalline (LC) monomers within matrix polymer solution to disperse ultra-fine particles of LCP within matrix polymer solution. After such insitu polymerization, solvent casting provided nanocomposites of commodity polymers as such PSt containing LCP particles. Thus, nano-composites were prepared with PSt-LC polyesters, ${ }^{3}$ PSt-LC polyamides, ${ }^{4}$ PStpolyimide, ${ }^{5}$ PSt-poly(L-glutamate) ${ }^{6}$ and PSt-Nylon $3 .{ }^{7}$

Mechanical properties of the nano-composites de-

${ }^{\dagger}$ To whom correspondence should be addressed. pended on size distribution of in-situ formed LCPs within matrix polymers. In studies on in-situ polymerization size distribution of the in-situ formed LCPs were controlled by solvents in which matrix polymers were dissolved to provide polymerization fields for dissolved LC monomers in solution. When a poor solvent for a matrix polymer was used, LCP particle size became large and with a good solvent for a matrix polymer, size of LCP particles was small.

Thus, dissolved LC monomers may form monomer clusters in solutions of matrix polymers and conformations of dissolved matrix polymers as random coil or extended chain structure in solution may influence domain size of dissolved LC monomers. Control of particle size and size distribution of PSt was thus carried out by insitu polymerization of St in the presence of matrix polymers in solution, as previously reported in a short communication. ${ }^{8}$ This paper describes results to verify this. Control of particle size of PSt by the in-situ polymerization is illustrated in Scheme 1 below:

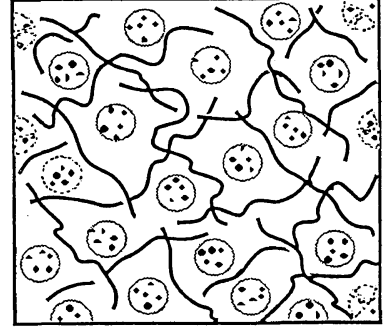

Good solvent : Ethanol Matrix Polymers Monomers

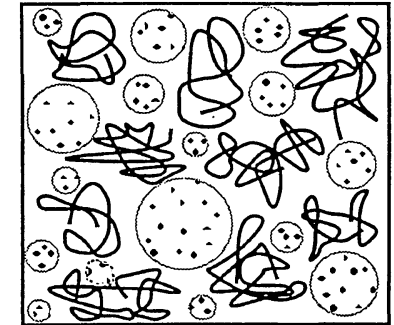

Poor solvent : $n$-Hexane

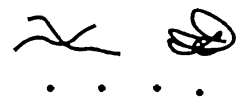

Scheme 1. 


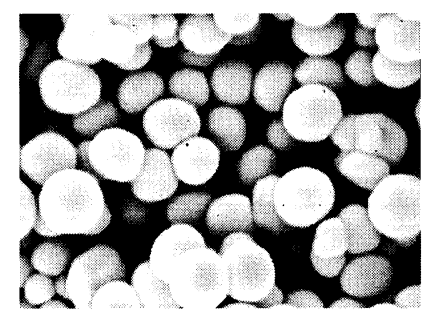

(a) Ethanol: $n-$ Hexane $=100: 0$

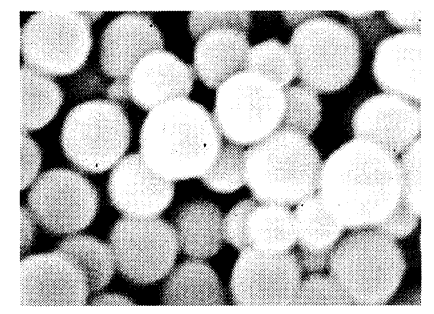

(d) $65: 35$

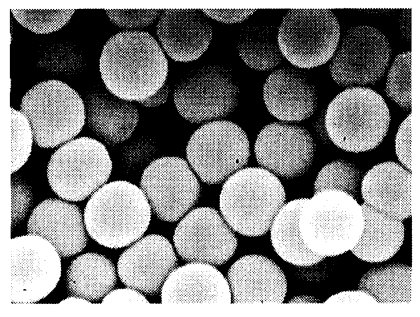

(b) $90: 10$

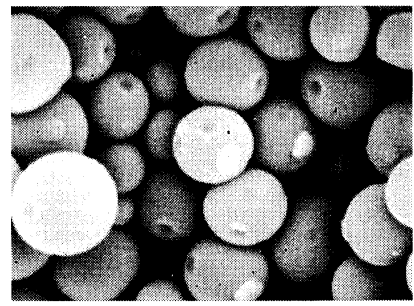

(e) $50: 50$

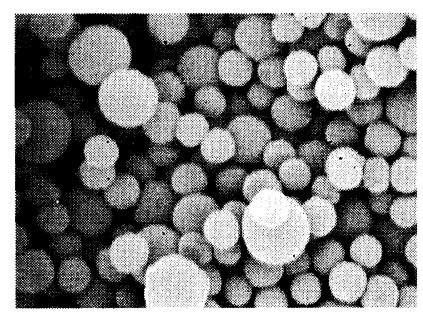

(c) $80: 20$

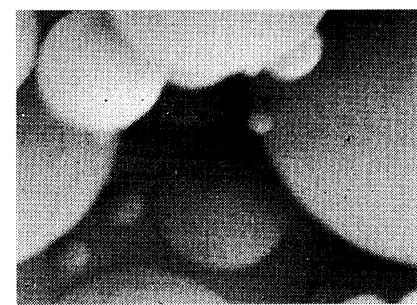

(f) $30: 70$

Figure 1. SEM pictures of PSt particles by in-situ polymerization in the presence of PMVE in mixed solvents of ethanol $/ n$-hexane.

\section{EXPERIMENTAL}

\section{Materials}

St and methylmethacrylate (MMA) were purified by washing with alkaline solution, followed by distillation. All solvents were purified by distillation. PMVE, poly(4vinylpyridion) (P-4 Vpy), poly(vinylpyrrolidone) (PVPy) of various molecular weights and poly(dimethylsiloxane) (PDMS) as matrix polymers were purchased from Wako Chemicals and were purified after reprecipitation either from ethanol or acetone and drying.

\section{In-situ Polymerization}

In-situ polymerization of St and other comonomers was carried out in mixed solution of poor-good solvents in the presence of matrix polymers using a radical initiator. When poly(methylvinylether) (PMVE) was used as a matrix polymer, $3 \mathrm{~g}$ PMVE were dissolved in $30 \mathrm{~g}$ mixed solvent of ethanol and $n$-hexane at various ratios, followed by adding $5 \mathrm{~g}$ St and $0.05 \mathrm{~g}$ azobisisobutyronitrile (AIBN) as radical initiator. No phase separation occurred and a clear solution was obtained. PSt did not dissolve in mixed solvents at mixing ratios up to $30 / 70$ (wt\%) of ethanol $/ n$-hexane. The entire solution was placed in a $200 \mathrm{~mL}$ three-necked flask and heated at 60 ${ }^{\circ} \mathrm{C}$ for $18 \mathrm{~h}$ under nitrogen atmosphere with stirring at $1500 \mathrm{rpm}$ by a propeller type mechanical stirrer. No effects on size or size distribution of PSt particles were found by changing stirring speed in the range of 1000 and $2000 \mathrm{rpm}$. No significant change of size and size distribution of PSt particles occurred when a reaction vessel of $500 \mathrm{~mL}$ three-necked flask was used.

After polymerization excess methanol was added to precipitate PSt particles separated by centrifuge, followed by washing with methanol and drying. Copolymerization of St and MMA was carried out in a similar way.

\section{Characterization}

Particle size and size distribution of PSt were determined by scanning electron microscopy, (Hitachi S-430). Molecular weight of polymers were determined in THF at $0.1 \mathrm{wt} \%$ using a Shimadzu C-R4AX GPC. Polymers were identified by NMR and FT-IR.

\section{Neutron Scattering}

Small angle neutron scattering of deuterated St in matrix polymer solutions was measured by cold neutron $(1=0.6 \mathrm{~nm})$ from a SANS-J neutron reactor of the Japan Atomic Energy Research Institute at Tokaimura. Deuterated St monomer (D-St) was dissolved in mixed solvents of ethanol $/ n$-hexane at various ratios under the same conditions as in-situ polymerization with PMVE (solvent/PMVE/D-St $=30 / 3 / 5$ in weight ratio) in the presence of AIBN. Small angle scattering was measured in a quartz cell $2 \mathrm{~mm}$ in thickness.

\section{RESULTS AND DISCUSSION}

\section{PMVE as a Matrix}

When in-situ polymerization of St was carried out in mixed solvents of ethanol $/ n$-hexane in the presence of PMVE of molecular weights of 46000 , initial clear transparent solution became turbid to form emulsion at the end of polymerization. Polymer particles were identified as pure PSt by NMR and FT-IR analyses. SEM pictures of PSt particles are shown in Figure 1 which indicates particle size increases with $n$-hexane, a poor solvent of PSt. Particle size distribution of PSt are shown in Figure 2 as function of mixing ratios of $n$-hexane to ethanol. In Figure 2 particle size distribution of PSt becomes broader with increasing mixing ration of $n$-hexane to ethanol. 

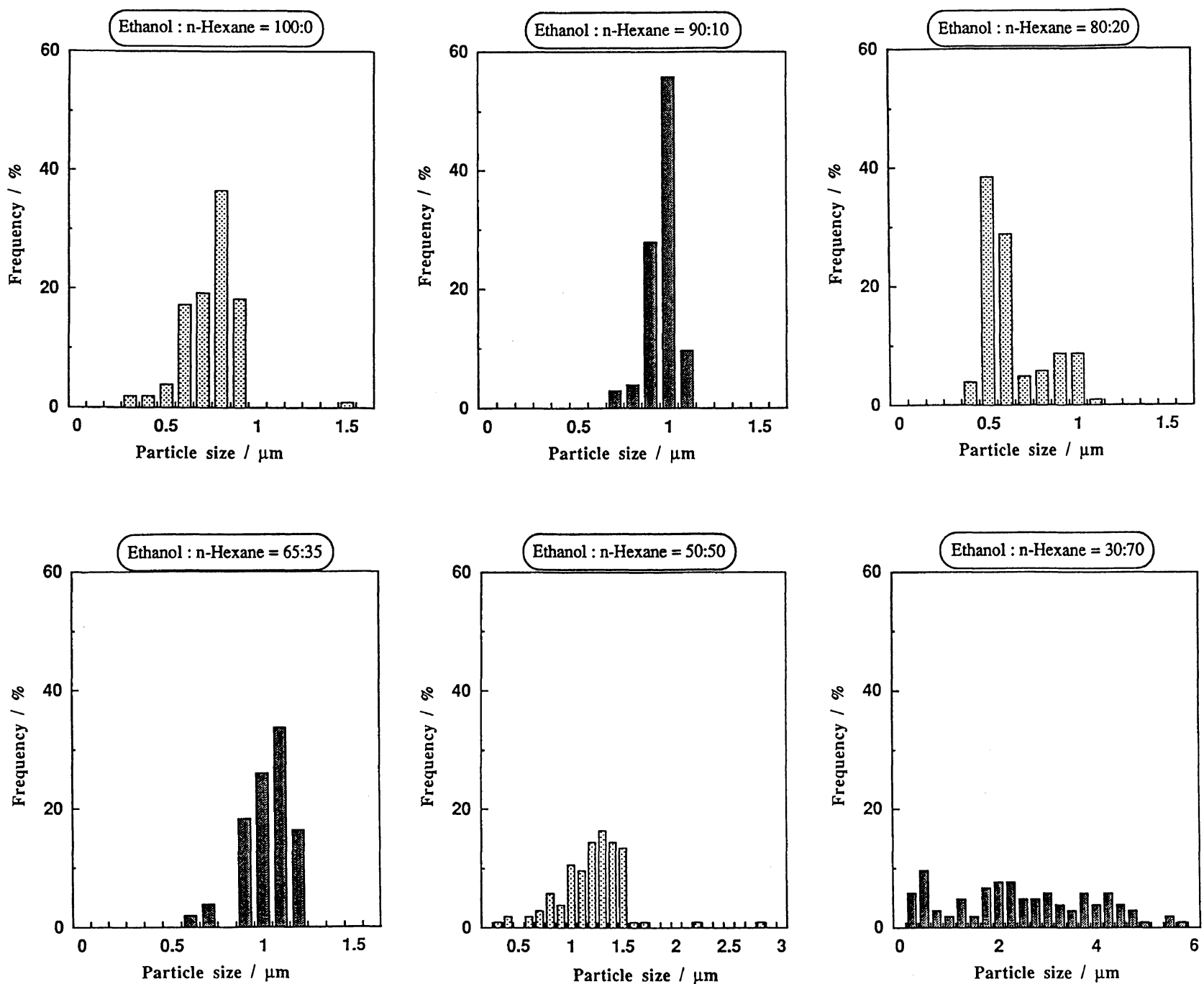

Figure 2. Particle size distribution of PSt obtained by in-situ polymerization in the presence of PMVE in mixed solvents of ethanol/nhexane.

Table I. Characterization of PSt particles obtained by in-situ polymerization in the presence of PMVE

\begin{tabular}{|c|c|c|c|c|c|c|c|}
\hline \multirow{2}{*}{$\begin{array}{c}\text { Sample } \\
\text { No. }\end{array}$} & \multicolumn{2}{|c|}{ Solvents } & \multirow{2}{*}{\multicolumn{2}{|c|}{$\begin{array}{c}M_{\mathrm{w}}^{\mathrm{a}} \\
\left(\times 10^{-4}\right) \\
\end{array}$}} & \multirow[b]{2}{*}{$M_{\mathrm{w}} / M_{\mathrm{n}}$} & \multicolumn{2}{|c|}{ Particle size } \\
\hline & Ethanol/g & $n$-Hexane/g & & & & $/ \mu \mathrm{m}$ & $\sigma \mathrm{m}$ \\
\hline 1 & 30.0 & 0.0 & 28 & 2.0 & 1.44 & - & - \\
\hline 2 & 27.0 & 3.0 & 25 & 2.0 & 1.46 & - & - \\
\hline 3 & 24.0 & 6.0 & 20 & 2.0 & 1.41 & - & - \\
\hline 4 & 19.5 & 10.5 & 33 & 1.9 & 1.46 & - & - \\
\hline 5 & 15.0 & 15.5 & 24 & 2.2 & 1.55 & - & - \\
\hline 6 & 9.0 & 21.0 & 12 & 2.0 & 1.57 & - & - \\
\hline 7 & 30.0 & 0.0 & 78 & 46.8 & 6.94 & 0.7 & 0.15 \\
\hline 8 & 27.0 & 3.0 & 90 & 49.9 & 9.57 & 1.0 & 0.08 \\
\hline 9 & 24.0 & 6.0 & 42 & 13.0 & 3.01 & 0.6 & 0.18 \\
\hline 10 & 19.5 & 10.5 & 38 & 4.4 & 2.16 & 1.0 & 0.13 \\
\hline 11 & 15.0 & 15.0 & 38 & 2.3 & 1.64 & 1.2 & 0.33 \\
\hline 12 & 9.0 & 21.0 & 28 & 2.6 & 1.59 & 2.5 & 1.44 \\
\hline
\end{tabular}

${ }^{\mathrm{a}}$ Determined by GPC method. Sample No. $1-6$ were polymerized with matrix polymer. Sample No. $7-12$ were polymerized with matrix polymer (PMVE).

When PMVE was absent in solution, a bulky solid of PSt was isolated adhered to the wall of the reactor. Results of the in-situ polymerization of St in the absence or presence of PMVE are summarized in Table I, which indicates mean particle size and size distribution increase with poor solvents of PSt as polymerization media.
The conformation of PMVE in mixed solution influence particle size and size distribution of PSt as the conformation of PMVE in mixed solution changes from extended to random coil structure, leading to size change of St monomer cluster in matrix polymer solution. Figure 3 shows mean particle size of PSt and solution vis- 


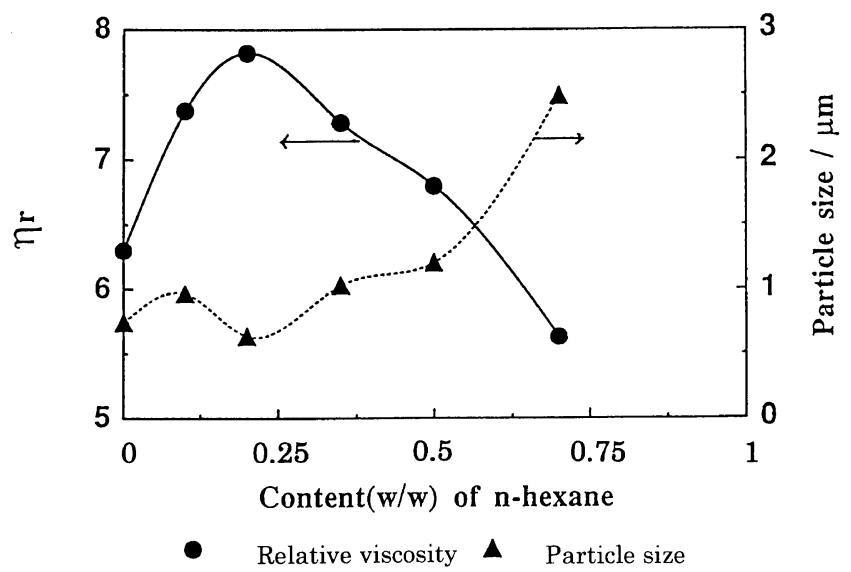

Figure 3. Relations between mean particle size of PSt and solution viscosity of PMVE solution.

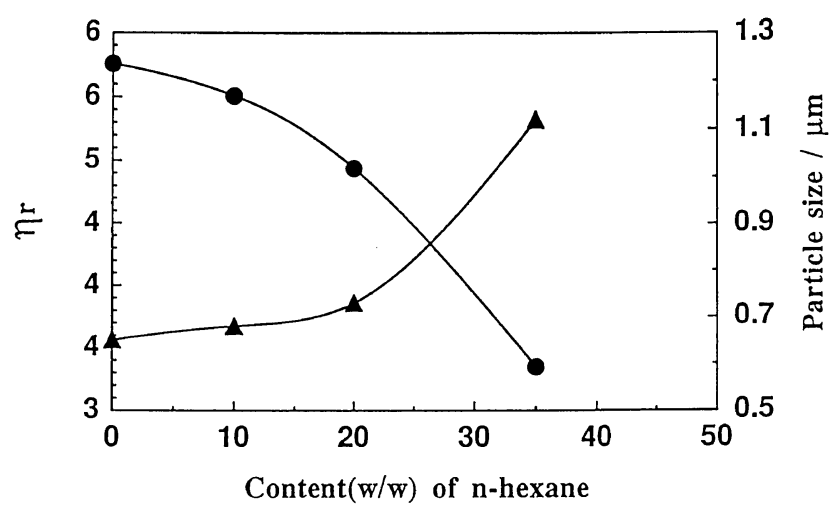

- Relative viscosity $\Delta$ Particle size

Figure 4. Relationships between solution viscosity of P-4-Vpy and particle size of PSt as function of $n$-hexane ratios.

Table II. Characterization of PSt particles obtained by in-situ polymerization in the presence of various concentrations of PMVE

\begin{tabular}{ccccccc}
\hline Sample & Concentration & & & \multicolumn{2}{c}{ Particle size } \\
\cline { 5 - 7 } No. & of PMVE/\% & Yield/\% & $M_{\mathrm{w}}{ }^{\mathrm{a}}$ & & \multicolumn{2}{c}{$\sigma \mathrm{m}$} \\
\hline 1 & 1 & 25.8 & 8.2 & 3.83 & 1.9 & 0.55 \\
2 & 3 & 41.6 & 9.4 & 3.04 & 1.2 & 0.10 \\
3 & 5 & 63.0 & 17.5 & 3.93 & 1.5 & 0.26 \\
4 & 10 & 42.0 & 13.0 & 3.10 & 0.6 & 0.18 \\
5 & 15 & 72.0 & 30.7 & 5.18 & 0.8 & 0.08 \\
6 & 20 & 86.0 & 27.6 & 3.77 & 0.8 & 0.08 \\
7 & 25 & 92.4 & 33.4 & 5.42 & 0.6 & 0.09 \\
\hline
\end{tabular}

${ }^{\text {a }}$ Determined by GPC method.

cosity of PMVE as function of $n$-hexane (wt ratios). Solution viscosity of PMVE reached maximum at $80 / 20$ ethanol and $n$-hexane. Mean particle size reached minimum at the same mixing ratios. This result may coincide with the conformational change of cluster size of St monomer in mixed solution of PMVE.

When the concentration of PMVE in mixed solution was changed from $1 \mathrm{wt} \%$ to $25 \mathrm{wt} \%$, particle size of resulting PSt decreased with concentration of PMVE. Insitu polymerization results are summarized in Table II. Mean particle size of PSt decreased, while yields and molecular weights of PSt increased with PMVE, presumably owing to the formation of St monomer cluster in PMVE solution, as illustrated in Scheme 1.

If in-situ polymerization of St proceeds through the formation of monomer clusters in matrix solution, copolymerization composition of St and MMA should be the same as bulk copolymerization of St and MMA when copolymerization of St and MMA is carried out in the presence of PMVE. Therefore, St and MMA were copolymerized at various molar ratios in the absence or presence of PMVE under the same conditions as St insitu polymerization. Copolymerization was stopped at low yield below $10 \mathrm{wt} \%$. Fine particles of the coplymers having mean particle size of $2 \mu \mathrm{m}$ were obtained in the presence of PMVE. However, no significant differences of copolymer composition were observed in bulk, solution and in-situ polymerization and monomer cluster formation during in-situ polymerization was not confirmed by copolymerization.

\section{P-4-Vpy as a Matrix}

P-4-Vpy of molecular weight of 277000 was used as matrix polymer. In-situ polymerization of St was carried out in the presence of P-4-Vpy in mixed solvents of ethanol and $n$-hexane. No phase separation occurred and a clear solution was obtained. As in-situ polymerization proceeded, PSt was obtained in an emulsion and SEM pictures of PSt indicated that particle size of PSt increased with $n$-hexane in mixed solvents. Relationships between solution viscosity of P-4-Vpy and particle size of PSt are shown in Figure 4, as function of $n$-hexane ratios in the mixed solvents, while particle size distribution of PSt is shown in Figure 5. PSt particle size increased and size distribution of PSt particles became broader with $n$-hexane, a poor solvent for PSt. These solvent effect coincide with the results of PMVE as matrix polymer.

\section{PVPy as a Matrix Polymer}

St was polymerized in ethanol $/ n$-hexane mixed solvents at various volume ratios in the presence of PVPy of molecular weight of 24000. No phase separation occurred for the solutions. In-situ polymerization conditions were the same as in the presence of PMVE. SEM pictures of PSt particles indicated particle size of PSt increase with $n$-hexane in mixed solvents. Particle size distribution became broader with $n$-hexane in mixed solvents, as shown in Figure 6 . These results were similar to those in the presence of PMVE as mentioned before.

The in-situ polymerization of St in the presence of PVPy of various molecular weights was carried out in mixed solvents of ethanol $/ n$-hexane at $80 / 20$ and 50/50. 

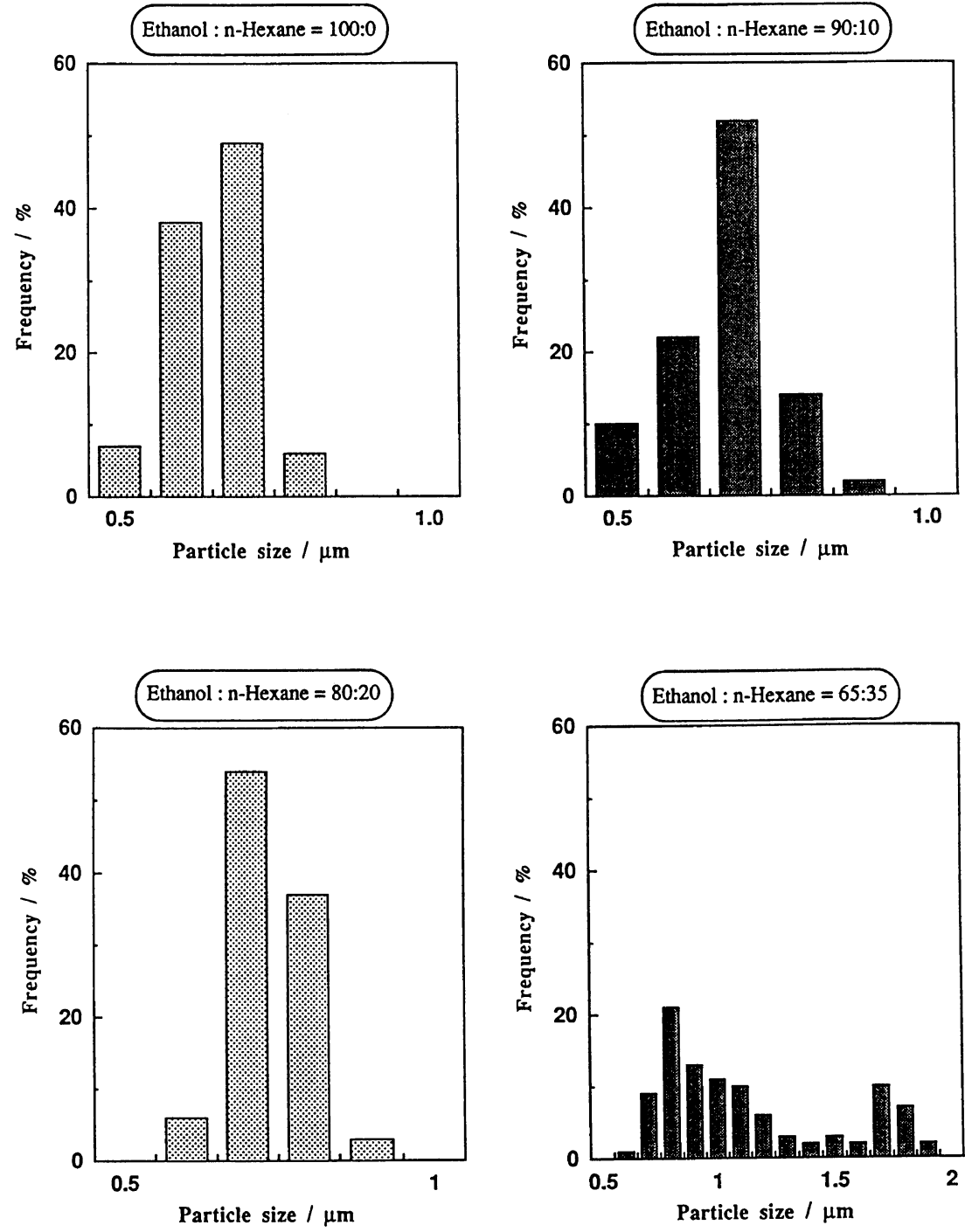

Figure 5. Size distribution of PSt particles obtained in the presence of PVPy.

SEM pictures of resulting PSt particles showed that particle size of PSt decreased with molecular weight of PVPy as matrix polymer in a mixed solvent of ethanol $/ n$ hexane $=80 / 20$, while no clear PSt paticle size for molecular weight of PVPy was observed in a mixed solvent of ethanol $/ n$-hexane $=50 / 50$. Mixed solvent of ethanol $/ n$ hexane $=50 / 50$ is rather a poor solvent for PVPy in which PVPy chain conformation may be random coils so that the formation of St monomer clustes would not be enhanced and in-situ polymerization may take place through simple solution polymerization to form irregular size PSt particles.

In-situ polymerization of St was carried out in a mixed solvent of ethanol $/ n$-hexane $=80 / 20$ and $50 / 50$ in the absence or presence of PVPy of molecular weight of 40000 , using various amounts of AIBN as radical initiator. Bulk polymerization of St was carried out under the same conditions to compare with in-situ polymerization. The results are summarized in Figure 7 which indicates that molecular weight dependency of resulting PSt obtained by the in-situ polymerization is close to that of bulk polymerization, when mixed solvent of ethanol $/ n$-hexane was 80/20. Molecular weight dependency of PSt obtained in a mixed solvent of ethanol $/ n$-hexane $=50 / 50$ in the presence of PVPy was close to solution polymerization in this solvent. These results strongly suggest the formation of St monomer cluster in matrix polymer solutions. Mixed solvent of $80 / 20$ of ethanol $/ n$-hexane is rather good solvent of PVPy dissolved in extended chain structures, while mixed solvent of $50 / 50$ of ethanol $/ n$-hexane is a poor solvent for PVPy to form random coil structures which would prevent the formation of St monomer cluster during in-situ polymerization, resulting in the same solution polymerization.

\section{PDMS as a Matrix Polymer}

Ethanol is a good solvent for matrix polymers such as PMVE, P-4-Vpy or PVPy, while $n$-hexane is a poor solvent. Since ethanol has less interaction with PSt, particle size control of PSt may be ascribed to the polarity effect of polymerization fields. Therefore, a reverse solvent effect was investigated in order to control particle size of PSt using PDMS as a matrix polymer for in-situ polymerization. In this case, $n$-hexane is a good solvent for PDMS, while ethanol is a poor solvent. In-situ polymerization of St in the presence of PDMS was carried out un- 

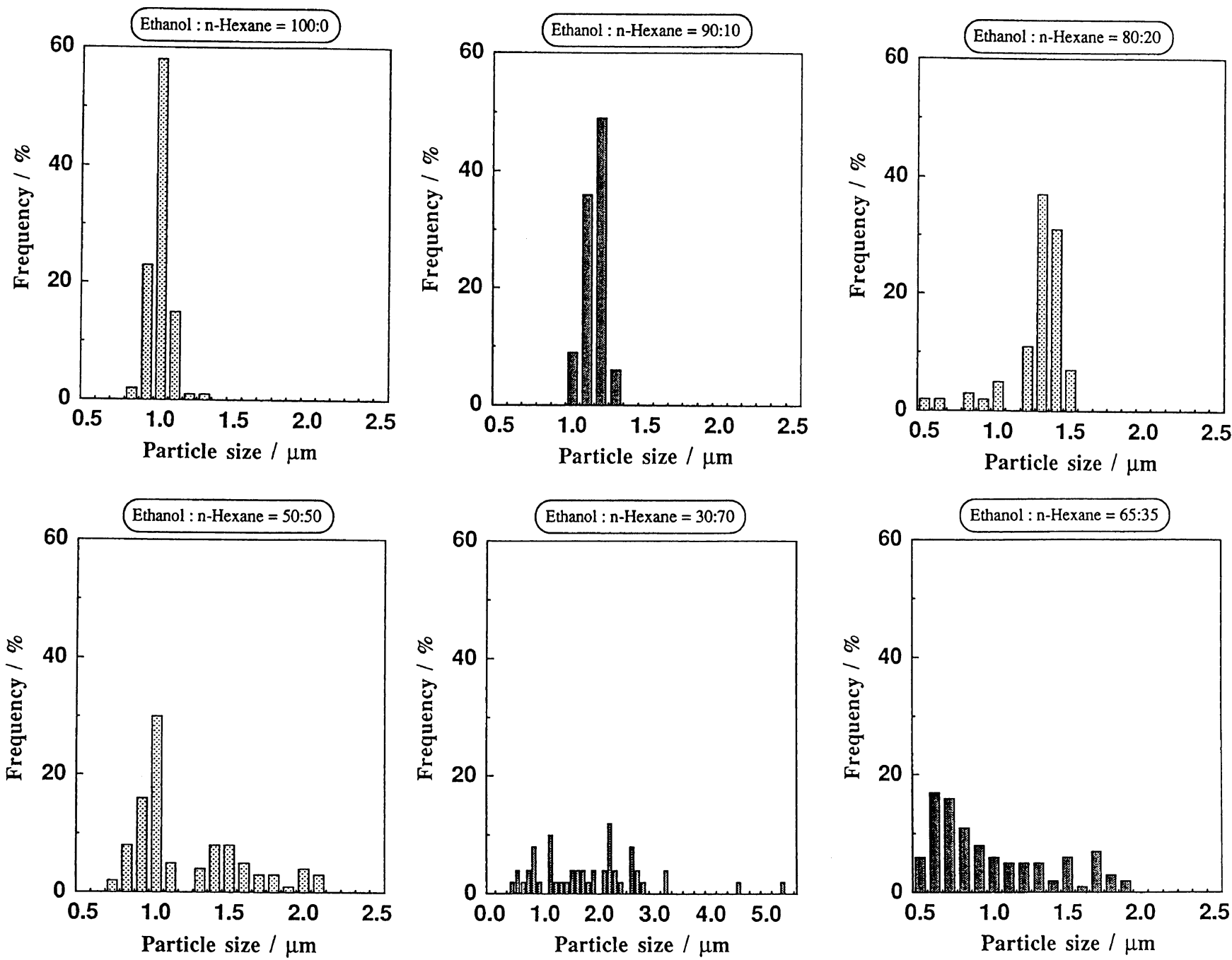

Figure 6. Size distribution of PSt particles in mixed solvents of ethanol $/ n$-hexane in the presence of PVPy.

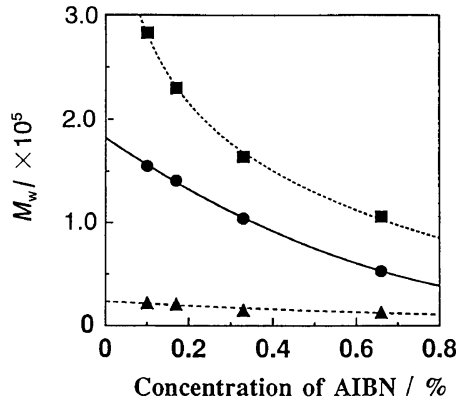

(a) Ethanol : n-Hexane $=8: 2$

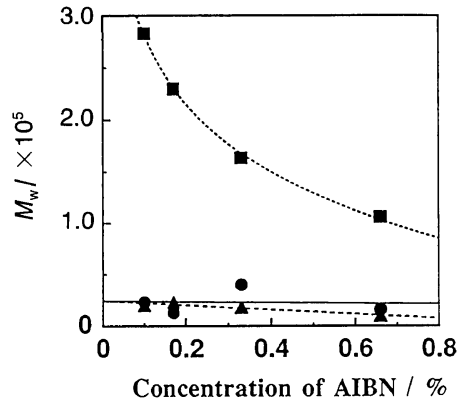

(b) Ethanol : n-Hexane $=5: 5$

- Bulk polymerization - Dispersion polymerization $\Delta$ Solution polymerization

Figure 7. Molecular weight dependency of PSt as functions of AIBN concentrations obtained by different methods.

der similar conditions as in the presence of PMVE.

No phase separation occurred for the solutions. Polymerization of St proceeded rather slowly in the presence of PMDS so that its time was adjusted to $48 \mathrm{~h}$ instead of $18 \mathrm{~h}$. A bulk solid of PSt was formed on the wall of the reactor and a small amount of a precipitation of PSt particles was observed. A stable emulsion of PSt was obtained. Particle size distribution of PSt in the emulsion as function of mixed ratios of ethanol $/ n$-hexane are summarized in Figure 8. Generally speaking, particle size and distribution became larger and broader with increasing ratios of ethanol in the mixed solvents, a poor solvent for PDMS. Yields of PSt ranged form 2 to $47 \%$ and were not reproducible, presumably owing to chain transfer reactions to PDMS during in-situ polymerization, since in-situ polymerization in the presence of 

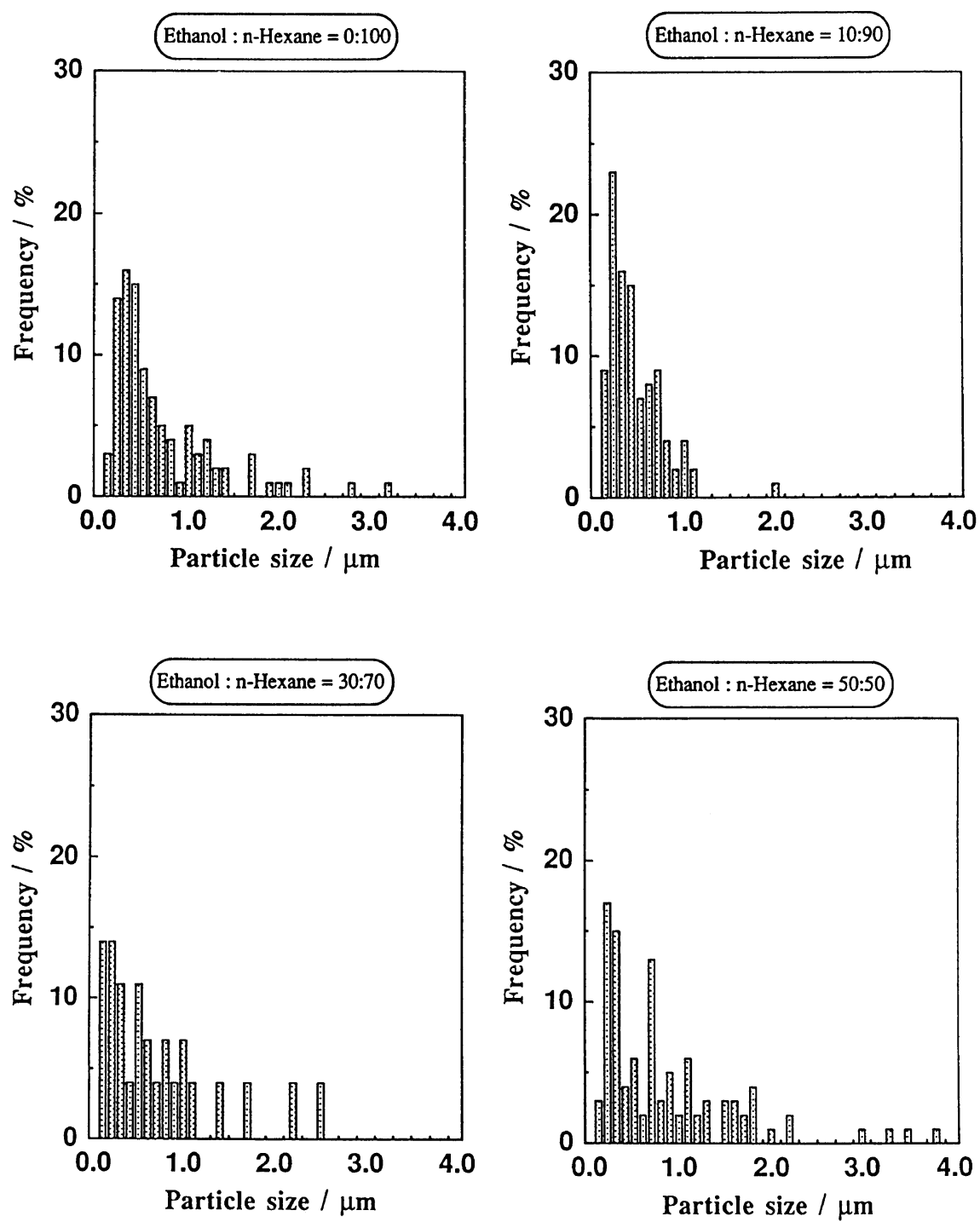

Figure 8. Size distribution of particles in mixed solvents of $n$-hexane/ethanol in the presence of PDMS.

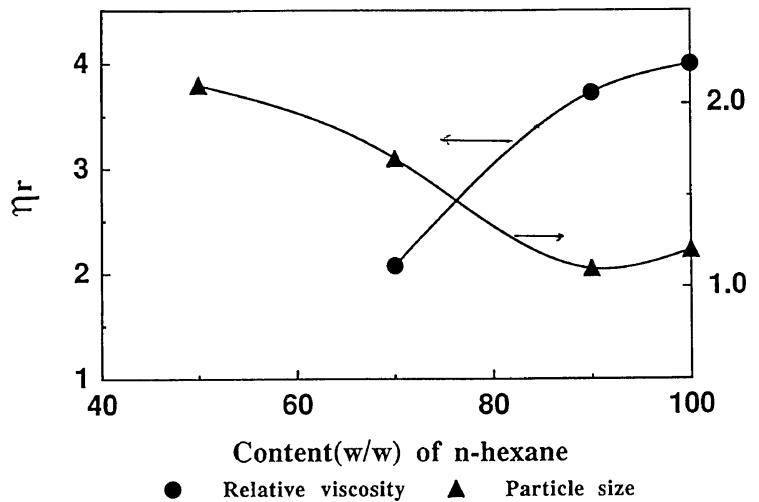

Figure 9. Relationships between solution viscosity of PDMS and mean particle sizes as function of $n$-hexane ratios.

PMDS took much longer than in the presence of PMVE, with the formation of bulk PSt mass.

Figure 9 indicates a relationship between solution viscosity of PDMS solutions and particle size, as function of $n$-hexane in mixed solvents. Mean particle size of PSt decreased with $n$-hexane in mixed solvents which led to decrease of solution viscosity. These results coincided with the results obtained for PMVE, P-4-Vpy, and PVPy. Increase of solution viscosity resulted in size decrease of PSt particles. Therefore, St monomer clusters may form in the PDMS solution as in the case of other matrix polymers, resulting in control of PSt particles by conformational change of PDMS in solution.

\section{St Monomer Cluster Detection by Neutron Scattering}

If St monomer clusters are formed in matrix polymer solution, it would be possible to detect clusters by small angle neutron scattering. ${ }^{9}$ Figure 10 indicates scattering profiles of small angle neutron scattering as function of time. Figure 10 also includes a scattering profile from PMVE in ethanol. It is seen in Figure 10 that a scattering of neutron derived from concentration gradients between ethanol and PMVE is observed as background. A scattering of neutron in a wave range of $q>0.5 \mathrm{~nm}^{-1}$ which is independent of wave number is derived from non-interfering scattering of exciting hydrogen in the system. When D-St monomer was added to matrix solution, a broad maximum peak was observed as shown in dark area between 0.1 and $0.3 \mathrm{~nm}^{-1}$. An apparent area 


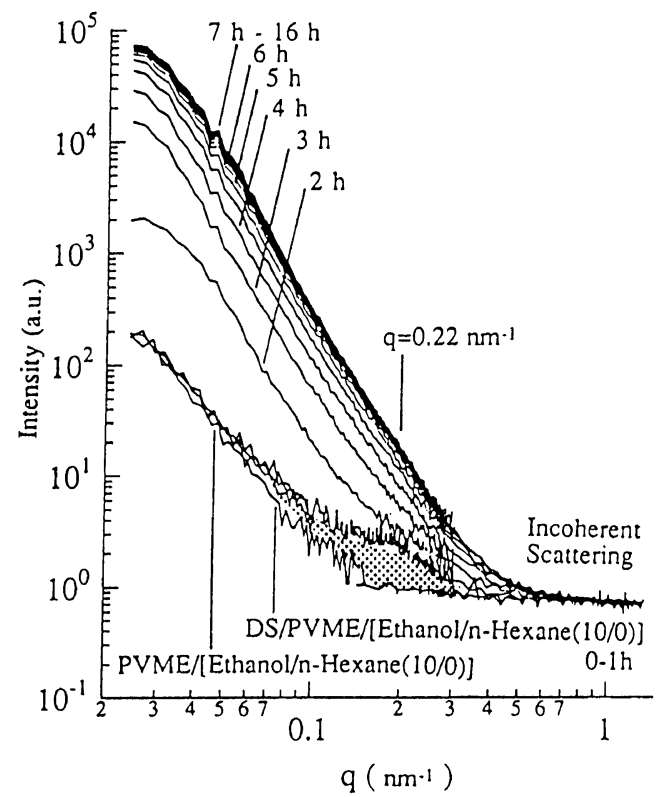

Figure 10. Neutron scattering profiles of D-St solution in the presence of PMVE as function of time.

size corresponding to the maximum was about $20 \mathrm{~nm}$ and the added D-St monomer may form a cluster in the matrix polymer solution.

Solutions were heated at $55^{\circ} \mathrm{C}$ to initiate the in-situ polymerization and neutron scattering was measured for $1 \mathrm{~h}$. No significant change of the scattering profile was observed, while at $2 \mathrm{~h}$, increase of scattering intensity was observed in the wave range of $q>0.3 \mathrm{~nm}^{-1}$. At this time, the solution became turbid and changed to emulsion. After $3 \mathrm{~h}$, scattering intensity increased in the wave number range to approach to almost constant scattering profile at $7 \mathrm{~h}$. These scattering profiles suggest the formation of D-St fine particles having a sharp interface with the matrix polymer solution after $2 \mathrm{~h}$. The scattering profile $I(q)$ in the Porod range may be approximately expressed as following equations:

$$
\begin{gathered}
I(q)=2 \pi \Delta B^{2}\left[S(t) / V_{0}\right] q^{-4} \exp \left(-\sigma(\tau)^{2} q^{2}\right) \\
d(t)=(2 \pi)^{0.5} \sigma(\tau)
\end{gathered}
$$

where $S(t)$ is total dimension of the interface in the system and increases with the growth of particles. $V_{0}$ is the volume of the system which is independent of polymerization time. $\Delta B$ is mean scattering length between the matrix polymer and D-St particles. $\sigma(t)$ may be expressed as eq 2 with relation to the characteristic thickness of interfaces between the matrix polymer and particles.

When $n$ is expressed as the number of particles, the radius $R(t)$ of particle be given as $S(t)=4 p n R(t)_{2}$. Assuming that particles $n$ formed at the initial stage is constant during in-situ polymerization, time change of scattering intensity can be attributed to increase of $R(t)$. Porod plots were carried out using scattering profiles below $q<0.22 \mathrm{~nm}^{-1}$, and relative dimensions of interfaces $S(t) / V_{0}$ and thickness of the characteristic interface $d(t)$

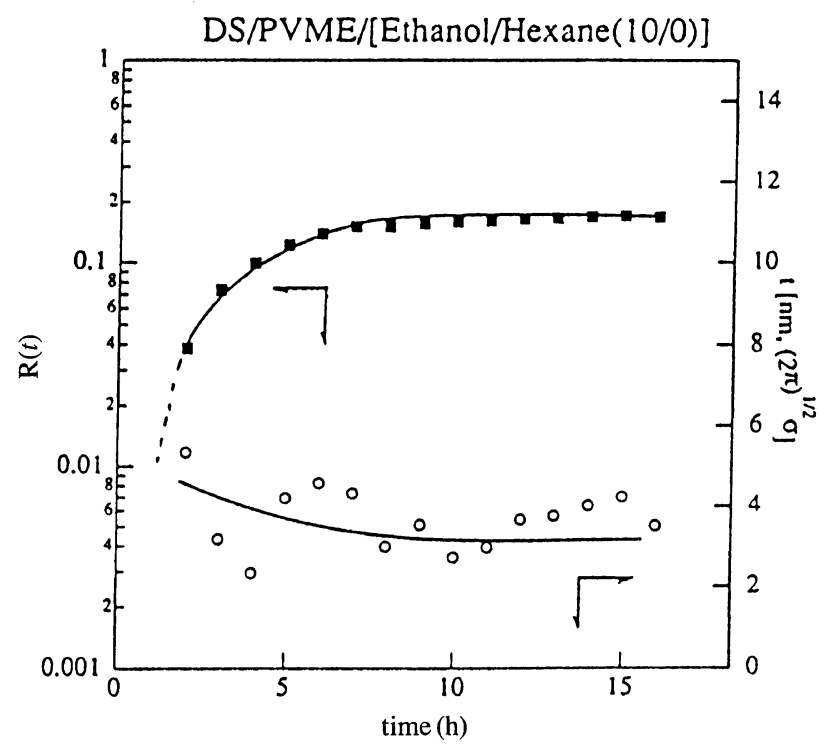

Figure 11. $R(t)$ and $d(t)$ changes as function of time.

were evaluated. Figure 11 indicates increase of $R(t)$ and $d(t)$ as function of polymerization time. These results show close agreement with experimental results on particle size growth during in-situ polymerization. This strongly supported by neutron scattering experiments that the St monomer cluster formation may occur during in-situ polymerization.

\section{CONCLUSION}

In-situ polymerization of St was carried out in mixed solvents of ethanol $/ n$-hexane in the presence of matrix polymers such as PMVE, P-4-Vpy, PVPy, and PDMS. Particles size and their distribution could be controlled by changing solvent composition from good to poor solvent systems, which influenced conformations of matrix polymers from extended to random coil structures in solution. Dissolved St monomer may thus form monomer clusters in the matrix polymer solutions and St monomer is not homogeneously dissolved. The formation of the St monomer cluster was supported by neutron scattering experiments.

\section{REFERENCES}

1. A. Ueno, in "Utra-fine Particles", C. Hayashi, R. Uyeda, and A. Tasaki, Ed., Noyes Publications, New Jersey, N. J., 1996, p 253.

2. Y. Almong, Br. Polym. J., 14, 131 (1982).

3. N. Ogata, K. Sanui, and H. Itaya, Polym. J., 22, 85 (1990).

4. E. Debeaupte, M. Watanabe, K. Sanui, and N. Ogata, Chem. Mater., 4, 1123 (1992).

5. A. K. Shu, E. Debeaupte, M. Watanabe, K. Sanui, and N. Ogata, New Polym. Mater., 3, 249 (1992).

6. Y. Ayaki, M. Rikukawa, M. Watanabe, K. Sanui, and N Ogata, Polym. J., 26, 325 (1994).

7. Y. Ann, M. Rikukawa, K. Sanui, and N. Ogata, Polym. J., 28, 496 (1996).

8. H. Chujo, M. Rikukawa, K. Sanui, and N. Ogata, Kobunshi Ronbunshu., 50, 349 (1993).

9. S. Saito, S. Koizumi, K. Matsuzaka, S. Suehiro, and T. Hashimoto, Macromolecules, 33, 2153 (2000). 